\title{
Medicina di Laboratorio: un futuro possibile
}

\author{
Laboratory Medicine: a look into the future
}

\author{
Piero Cappelletti
}

Ricevuto: 16 luglio 2012 / Accettato: 25 luglio 2012

(C) Springer 2012

Riassunto La trasformazione del Laboratorio e della Medicina di Laboratorio è sconvolgente: entrambi aprono incredibili possibilità diagnostiche, ma rischiano di far scomparire disciplina e professionisti. Tutte le previsioni danno per inarrestabile il progresso verso una medicina più "precisa" e focalizzata al "nuovo" paziente. Le organizzazioni del Laboratorio, così come le abbiamo conosciute e abbiamo tentato di difendere e far maturare attraverso dinamiche evolutive, non sono più adeguate alle nuove sfide della sanità. I professionisti della Medicina di Laboratorio, la loro educazione istituzionale e continua, $\mathrm{i}$ contenuti professionali di cui dispongono sono sottoposti a radicali innovazioni. Si tratta di immaginare il futuro con coraggio e con la prospettiva di saper cambiare profondamente i nostri saperi e i nostri modi di operare. Molte suggestioni degli autori citati in questo Editoriale possono essere utili. Una riflessione aperta e approfondita su ruoli e scenari al tempo della crisi è necessaria. Alcuni strumenti concettuali e operativi per affrontare il futuro, tuttavia, sono stati già stati definiti nel "Patto per la Modernizzazione e l'Umanizzazione della Medicina di

P. Cappelletti (ه)

IRCCS CRO Aviano

Via Franco Gallini 2, 33081 Aviano (PN), Italy

E-mail: piero.cappelletti@cro.it
Laboratorio in Italia", che mantiene ancora oggi il suo valore di visione e di proposta.

Parole chiave Medicina di Laboratorio - automazione · information technology $\cdot$ innovazione distruttiva

Summary The transformation of Clinical Laboratory and Laboratory Medicine is breathtaking, full of opportunities cleverly disguised as challenges but potentially disruptive of discipline and professionals. All predictions see as unstoppable the progression toward "precision" medicine focused to a "new" (out)patient. The traditional organization of Clinical Laboratory, modified in past years by a dynamic stability changing, is no longer fit to the challenges of "new" healthcare systems. A disruptive innovation is expected for Laboratory professionals, their school and continuous education, and their professional's knowledge and skills. There is a need to imagine a future for Laboratory Medicine, with courage and awareness of the radical changing of knowledge and roles. Many suggestions from authors cited in this Editorial can help, but some conceptual and operational tools were already defined some time ago in the "Pact for Modernization and Humanization of the Laboratory Medicine in Italy”, that maintains its value as vision and proposal for the future of Laboratory Medicine.

Key words Laboratory Medicine · automation · information technology $\cdot$ disruptive innovation 


\section{Introduzione}

La trasformazione della Medicina di Laboratorio nel ventunesimo secolo risente, come ricordano Parker e Talbert [1], dell'evoluzione culturale del Mondo, con l'irrompere del "soggetto" postmoderno nell' arena filosofica e sociale [2] e dello sviluppo dell'organizzazione sociale della Medicina, centrata sul paziente-cittadino, ma attanagliata dal problema del suo costo esponenzialmente crescente [3].

In questo Editoriale si cercherà di descrivere come si può immaginare il futuro della Medicina di Laboratorio, sulla base dei percorsi culturali e tecnologici degli ultimi decenni. La richiesta di esami di laboratorio aumenta progressivamente insieme con l'aspettativa di una sempre maggiore affidabilità, velocità, sicurezza e convenienza, in una molteplicità di situazioni cliniche (prevenzione, diagnosi, monitoraggio, ma anche check-up o medicina del desiderio) e con una dispersione di modalità analitiche [self-testing, point-of-care testing (POCT) in tutte le accezioni, ambulatorietà, ricovero], caratterizzate dalla disponibilità e facilità di accesso di test e metodi anche particolarmente delicati come quelli genetici. Ciò determina, da un lato, il diffondersi del concetto degli esami di laboratorio come "merce" (commoditization), ma, dall'altro, almeno in un certo numero di casi, necessità e capacità interpretative non ristrette alle forme tradizionalmente utilizzate, ma di più pregnante contenuto informativo (precision medicine) [4]. I mezzi operativi per un'adeguata risposta alle esigenze cliniche e sociali si moltiplicano e frammentano e possono essere governati solo attraverso un'efficiente e innovativa information and communication technology (ICT) e una visione patient-centered del sistema sanitario e della rete laboratoristica da parte dei suoi leader e addetti.

\section{Com'è cambiato il Laboratorio}

Il Laboratorio clinico è stato ed è, molto probabilmente, l'ambiente più dinamico dell'intero scenario dell'applicazione dei risultati della ricerca alla medicina pratica [5]. Nella seconda metà del secolo scorso, l'avvento delle prime automazioni, il crescere della richiesta di esami, anche per il mutato clima sociale attento alla prevenzione e agli screening, e l'emergere di norme specifiche per la configurazione del Laboratorio e per le caratteristiche dei lavoratori coinvolti definiscono un primo cambiamento, per il quale il Laboratorio si rende autonomo e diviene più grande, organizzato, suddiviso in sofisticati settori e con personale "specialista". La suddivisione in "settori" era dettata da motivazioni disciplinari (ematologia, microbiologia), oppure da motivazioni puramente tecniche [immunometria, radio immuno assays (RIA)], talora dal combinarsi delle due (elettroforesi/proteine). Tuttavia, negli anni '90 del secolo scorso il panorama cambia. La proposta di nuovi test diagnosticamente efficaci è un continuum che non conosce rallentamenti. Ricordiamo, ad esemplificazione, l'emergere di nuovi agenti infettivi come Helicobacter pylori, la diffusione dei metodi di biologia molecolare in campo infettivologico, la moltiplicazione dell'uso e della tipologia di marcatori di malattia utili per la diagnosi (marcatori cardiaci) o la terapia [emoglobina glicosilata $\left(\mathrm{HbA}_{1 \mathrm{c}}\right)$ ], l'analisi genetica delle malattie mendeliane, di quelle neoplastiche e di alcune malattie "comuni" e tutta la prospettiva della ricerca traslazionale sui biomarcatori. Parallela a questo processo è l'introduzione in laboratorio di strumentazione e di sistemi analitici sempre più sofisticati, idonei a portare la specificità e la sensibilità dei test al massimo livello di ottimizzazione: la "rivoluzione tecnologica" che si esplicita compiutamente negli anni '90 del secolo scorso consiste nel progredire della tecnologia della misura, dell'automazione e robotizzazione, della tecnologia digitale e di comunicazione (internet e intranet) e della tecnologia del trasporto. In terzo luogo, il progressivo incremento dei costi totali, la modifica dei sistemi di rimborso/finanziamento e dell'organizzazione dei sistemi sanitari sia privatistici sia pubblicistici, da un lato, e la richiesta di diagnostica in tempo reale, dall'altro, mostrano i limiti del Laboratorio "compartimentato" e la necessità di un'organizzazione focalizzata al turnaround time (TAT). Tradizionalmente l'attività del Laboratorio è stata pensata come un percorso produttivo lineare tra l'input della richiesta e dei campioni biologici e l'output della risposta, con al centro una fase poco visibile e compresa dell'attività strettamente analitica (black box). Tuttavia, la rivoluzione organizzativa degli anni '90 del secolo scorso produce la nascita del concetto del riarrangiamento dell'organizzazione lineare del lavoro del Laboratorio in un percorso a "loop" e il concetto del riassemblamento delle dotazioni tecniche, separando le convenienze analitiche dalle competenze disciplinari. Un'importante conseguenza è il disaccoppiamento del rapporto cliente/fornitore in Medicina di Laboratorio, che acuisce la percezione di lontananza (e di non essenzialità) della disciplina rispetto alla cura del paziente [6].

Nel 2000, in un articolo dal significativo titolo Laboratory Automation: Traiectory, Technology, and Tactics, Markin e Whalen [7] puntualizzano il ruolo dell'automazione nel Laboratorio Clinico al passaggio del millennio (le 3T). Traiettorie. L'evoluzione del Laboratorio riflette i cambiamenti sociali e sanitari con le collegate necessità di contenimento dei costi e di ottimizzazione degli outcomes. Gli aspetti preminenti sono: 1) la riorganizzazione della cura intorno al paziente cronico 
(primary care, medicina del territorio) con il consolidamento e la diminuzione delle strutture ospedaliere; 2) il trasferimento alla pratica medica dei portati della ricerca scientifica: la "personalizzazione" deve essere intesa non solo sul fronte medico della diagnosi e della terapia, ma anche su quello più vasto assistenziale delle cure; 3) la demistificazione della medicina tradizionalmente intesa e l'utilizzo anche della moderna tecnologia della comunicazione per il coinvolgimento del paziente [8]. Tattiche. Molte sono le strategie di sopravvivenza applicate dai Laboratori in questa fase di cambiamento: esse si basano fondamentalmente sull'aumento della produttività e sul contenimento dei costi per test. Secondo Kiechle e Main [9], i metodi per aumentare il volume dei test sono l'allargamento del servizio esterno (attraverso contratti, joint-venture, nuovi menù e regionalizzazioni) e il consolidamento geografico dei laboratori, mentre i metodi per diminuire i costi sono l'automazione e la decentralizzazione, la diversificazione dello staff tecnico e la riduzione dei tempi (in particolare di degenza ma dei TAT in generale). Tecnologie. Il ruolo di fondamentale fattore di cambiamento della "rivoluzione tecnologica" sotto il profilo dei sistemi di misura, l'integrazione di molte funzioni analitiche in un unico strumento e la front-end automation, la miniaturizzazione dei sensori, dei componenti meccanici, di trasmissione ed elettronici (microchip) e, sotto il profilo della tecnologia digitale, i progressi hardware, software e delle periferiche dei computer e la loro integrazione in reti locali (local-area network, LAN) ed estese (wide-area network, WAN) - è stato magistralmente descritto da Burtis [10]. Il principale obiettivo dell'automazione e in particolare dell'automazione della fase preanalitica è eseguire in modo meccanico attività umane noiose, sporche e pericolose $\mathrm{o}$, come dicono gli americani, le $3 \mathrm{D}$ tasks: dull, dirty, dan- gerous. Ciò si risolve, quando sapientemente controllato da software adeguati [sistema di automazione di laboratorio (laboratory automation system, LAS)], in una standardizzazione dei processi e in un abbattimento della difettosità del sistema di molte decine di volte. La riorganizzazione del lavoro conduce a risparmio di personale, anche di molte unità tempo pieno equivalenti (full-time equivalent, FTE), risparmio di provette e di spazi. Il risparmio economico complessivo dovrebbe essere riutilizzato almeno in parte per nuove diagnostiche emergenti. Il personale risparmiato, almeno in parte, dovrebbe essere re-impiegato in attività di più alto valore aggiunto. Nell'articolo Concepts for the third generation of laboratory systems, Hoffmann [11] tratteggia la storia dell'automazione nel Laboratorio clinico in 3 tappe o generazioni successive di LAS: la comparsa dei "kit", gli strumenti analitici automatici, il consolidamento e l'integrazione strumentali nella prospettiva della total laboratory automation (TLA). David O'Brain, direttore dell'automazione allo Smith Kline Beecham, nell'esaminare il potenziale mercato di TLA negli Stati Uniti, alla fine degli anni ' 90 , ha evidenziato che su 5200 laboratori presenti solo 441 sarebbero stati in condizione di acquistare e gestire una TLA [12]. Oggi si parla di una $4^{\text {a }}$ generazione di LAS, caratterizzata da un governo informatico completamente bidirezionale, dalla gestione di tutte le provette con trasporto individuale, da un tempo di transito minore di 15 minuti per campione, da un'organizzazione modulare scalabile (caratterizzata da un point-ofspace sampling, da un'interfaccia bidirezionale con il sistema informatico di laboratorio (LIS), un disegno architettonico "lean") e da una possibilità di stoccaggio e recupero dei campioni in linea. Gli standard per un'adeguata automazione (LAS) sono definiti dal Clinical and Laboratory Standards Institute (CLSI).

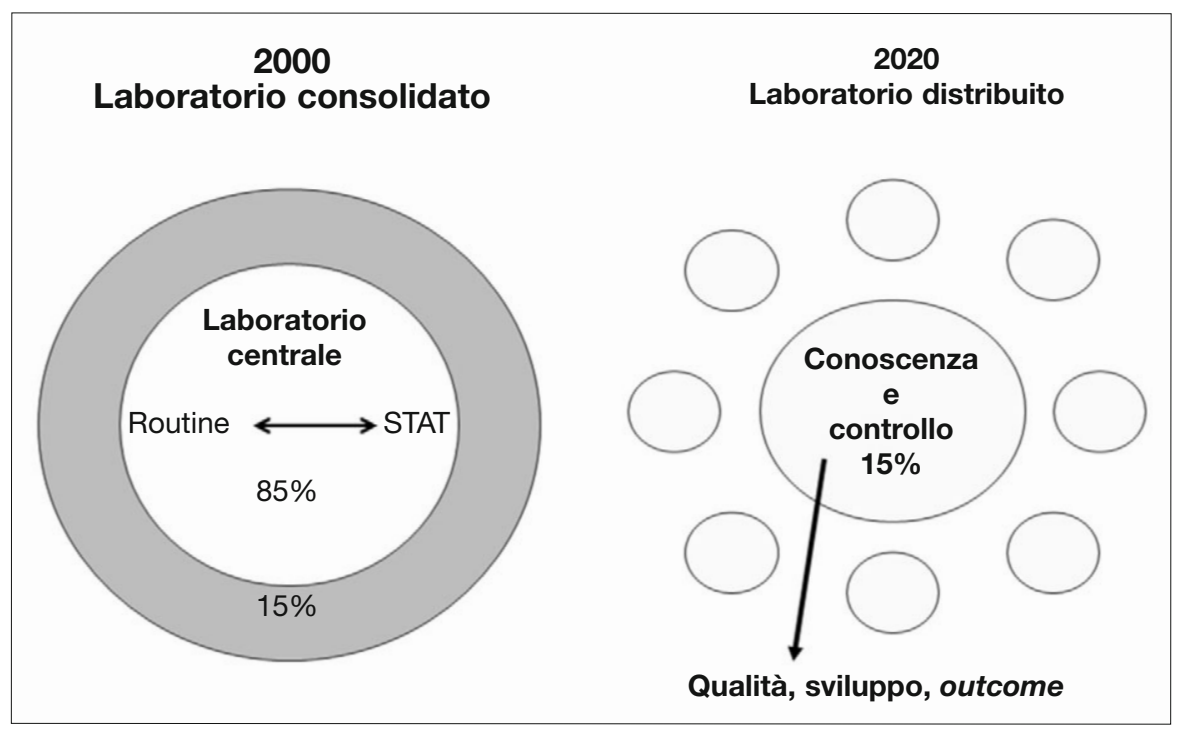

Fig. 1 Realizzazione del Laboratorio distribuito: il Laboratorio centrale mantiene le funzioni di knowledge management, di controllo della qualità e di governance, con le quali determina la produzione del servizio prevalentemente in periferia, la sua qualità, il suo sviluppo e la verifica degli outcomes clinici e assistenziali. STAT, short turnaround time. 
Sotto la spinta della nuova diagnostica e della rivoluzione tecnologica, delle aumentate aspettative individuali e sociali dalla medicina (legate ai cambiamenti demografici ed epidemiologici, ma anche alla percezione del potere salvifico della scienza) e della pressione per contenere costi in esponenziale continuo aumento, si evidenziano, dunque, due trend contrapposti in Medicina di Laboratorio. Da un lato la risposta dei Laboratori alla diagnostica urgente conosce una curva evolutiva, dalla dispersione all'interno del Laboratorio all'autonomia di un settore e quindi di nuovo all'integrazione. Negli anni 2000 la pressione per il decremento del TAT e la disponibilità di adeguati supporti tecnologici spingono la diffusione di strumenti di POCT - near patient testing (NPT) nel Regno Unito. Al contrario, forze centripete, di tipo fondamentalmente economico, premono per la realizzazione di un Laboratorio core fortemente automatizzato, contornato da Laboratori a risposta rapida e Laboratori satellite specialistici e, successivamente, per concentrazioni in Laboratori "regionali". Nascono Laboratori geograficamente "consolidati" (la risposta "americana", figlia della managed care) o reti di Diagnostica di Laboratorio (la risposta "britannica" della Pathology Modernisation) [6] e non solo (in quanto si tende a integrare diversi mezzi diagnostici), in quello che è definito distributed laboratory e, per gli aspetti organizzativi e di controllo, virtual laboratory. In questa nuova organizzazione del Laboratorio ci si attende la migrazione dell' $85 \%$ della diagnostica in siti periferici, non solo POCT, ma soprattutto self testing (Fig. 1).

\section{Forza e debolezza, opportunità e paura}

Dalla fine del secolo scorso, quando la rivoluzione organizzativa era già in corso e si affacciava sulla sponda europea dell'Atlantico, molti articoli hanno affrontato il tema del futuro della Medicina di Laboratorio, sotto il profilo della strutturazione dei Laboratori, delle politiche sanitarie a essa collegate, della tipologia e modalità di fornitura del servizio, delle conseguenze sulla disciplina e sui suoi professionisti $[6,8,13-17]$ e hanno valutato lo stato di avanzamento delle riorganizzazioni negli Stati Uniti [18], nel Regno Unito [19], nelle aree europee [20] e in Italia [21]. L'insieme delle sensibilità rappresentate negli articoli consente di sintetizzare alcune visioni comuni: l'ineluttabilità (e il fascino) del cambiamento insieme con il timore che megalaboratori e diminuzione/invecchiamento degli addetti conducano alla fine della disciplina e alla definitiva trasformazione in "merce" dell'esame di laboratorio; consapevolezza di necessarie trasformazioni strutturali e organizzative, spinte dalla tecnologia, che vanno possibilmente governate (il tema della qualità e della sua certificazione), ma che possono/devono condurre al superamento dei confini disciplinari e professionali tradizionali; ricerca delle possibilità di dimostrare il valore aggiunto della Diagnostica di Laboratorio, con la riscoperta del "referto", l'impegno al lavoro di comunicazione all'interfaccia clinica-laboratorio e gli studi di outcome, alla luce di una sempre più accettata EBLM (evidence-based laboratory medicine). Tuttavia, il clima diffuso è ben spiegato nella presentazione di un articolo dal significativo titolo "The future for laboratory medicine" pubblicato su The Bulletin of The Royal College of Pathologists nel 2010: "Some of us might be forgiven for wondering whether lab medicine has a future at all. However, some of us are confusing whether laboratory medicine in general has a future, with whether my laboratory or my specialty has a future"[22].

$\mathrm{Nel}$ 2006, O'Leary [23] ha effettuato un'analisi SWOT (strengths, weaknesses, opportunities, threats) molto interessante del moderno Laboratorio Clinico in area anglosassone. Secondo l'autore i principali punti di forza del Laboratorio sono: la presenza di uno staff dirigenziale giovane e motivato; ottimi standard di pratica con specifica tensione all'eccellenza, al confronto e all'accreditamento; capacità attrattive della Medicina di Laboratorio con opportunità educative e di crescita professionale; possibilità di ricevere fondi per la ricerca; prospettive di sviluppo tecnologico, specialmente per le biotecnologie. I punti di debolezza sono la crescita anno dopo anno delle richieste di impegno lavorativo con bassi investimenti strategici; sempre minor tempo per pensare allo sviluppo strategico, soffocati dall'incrementale routine; la battaglia contro inappropiatezze nella richiesta e nell'utilizzo delle risposte dei test; la decimazione dello staff; un'inadeguata politica di finanziamento, specie per le infrastrutture; la consapevolezza della "lontananza" dalla realtà clinica e della percezione della Medicina di Laboratorio come "invisibile" e "incompresa", anche a causa degli stessi operatori del Laboratorio. Le opportunità sono considerate enormi in relazione sia ai progressi della diagnostica e alla sua pervasività nella medicina contemporanea, sia alla scoperta dei punti specifici ove si crea il valore aggiunto del "prodotto" del Laboratorio, in rapporto alle prospettive della medicina personalizzata, dei network di ricerca e dei ruoli nella gestione della ristrutturazione dell'organizzazione della Medicina di Laboratorio. I rischi sono quelli della marginalizzazione nelle decisioni, dell'invisibilità, della frammentazione da un lato e dell'erosione dall'altro dei contenuti disciplinari e professionali, nella mancanza di un'adeguata analisi e ri-costruzione di ruoli e poteri della disciplina e dei suoi addetti.

È interessante confrontare questa analisi con quella condotta in Italia qualche anno prima con metodo 
Metaplan ${ }^{\circledR}$ [24]. I punti di forza sono la garanzia di qualità analitica (24\%), il rapporto con la clinica e l'appropriatezza $(33 \%)$, le capacità organizzative e gestionali (38\%), che riflettono il passato e il presente della disciplina. I flash (punti di contraddizione) sono relativi ai nuovi compiti: la consulenza diagnostica e la gestione delle risorse. La debolezza principale è la definizione dell'autorevolezza del ruolo (63\%) e i problemi individuati sono, conseguentemente, la visibilità del medico di laboratorio e una certa carenza di visione delle strategie future. Le opportunità da cogliere sono, da un lato, l'EBLM, l'appropriatezza e la consulenza e, dall'altro, l'entusiasmo delle novità diagnostiche. I flash sono relativi al terzo punto, le novità organizzative, segnatamente la contraddittorietà sul POCT e le problematiche emergenti dai nuovi ruoli professionali. I rischi paventati sono le riorganizzazioni selvagge (39\%) legate a una politica avvertita come distorta, maligna, improntata esclusivamente ai tagli (48\%), ma anche una preoccupazione per la sfiducia e i problemi che tormentano i professionisti. I vincoli avvertiti sono fondamentalmente le politiche sanitarie e le loro applicazioni, ma anche l'attività degli amministratori/amministrativi, la ripartizione delle risorse e degli pseudobudget e la pressione dell'industria. Le possibili sinergie con l'industria sono identificate in strategie coordinate per l'organizzazione sanitaria, in ricerca e sviluppo (trials clinico-diagnostici) e in attività formativa ed EBLM. I flash qui evidenziano i pericoli della lobby: confusione di ruoli e conflitto di interessi.

\section{Nel 2026 (o anche prima)}

Secondo alcuni autori [25] il futuro del Laboratorio è una total laboratory solution, un Laboratorio e-business, collaborazione verticale di meta-network tenuta insieme dall'evoluzione dell'information technology (IT). Secondo altri autori [26] il vero futuro è un hybrid laboratory basato sull'integrazione di esami in vitro, ex vivo e in vivo che integra monitoraggi classici in laboratorio con monitoraggi continui con biosensori interni o esterni. In effetti, progressivamente l'IT ha assunto un ruolo essenziale per il Laboratorio e ne è diventata veramente il sistema nervoso, in condizione di connettere tutte le sue parti, farle funzionare, avvertirne i messaggi, elaborare i dati in conoscenze. Sistemi informatici di laboratorio completamente integrati sono un pre-requisito per fornire un servizio clinico efficiente e per gestire il Laboratorio. A tal fine essi devono rispondere ai quattro livelli definiti dalla gestione operativa, dei dati, delle informazioni aggregate e della conoscenza, consentendo di registrare tutte le richieste per tutti i test, di tenere collegati on-line e in real-time tutti gli strumenti analitici, di registrare e valutare in tempo reale i dati del controllo di qualità, di validare automaticamente i risultati, di rilasciare elettronicamente i risultati agli utilizzatori clinici, di implementare sistemi di supporto alla diagnosi per migliorare gli output clinici e di supportare gli interventi all'interfaccia clinica-laboratorio dei gruppi multidisciplinari come audit, gestione del rischio clinico, controllo della patologia ed epidemiologia. Il CLSI provvede a una tra le più complete standardizzazioni del LIS. Poiché la funzionalità dell'IT dovrebbe essere determinata dalle necessità del servizio piuttosto che determinarne l'organizzazione (come ancora oggi continua ad avvenire), i LIS devono spostare il loro fuoco dai tradizionali problemi interni al Laboratorio, che sono ampiamente studiati e standardizzati e che dovrebbero essere definitivamente acquisiti, a quelli del servizio esterno, tenendo conto delle principali tendenze dei sistemi sanitari: consolidamento geografico dei laboratori, aumentato ruolo della Medicina primaria, decentralizzazione della diagnostica presso i luoghi dell'assistenza e della cura, necessità di un maggiore impatto dell' appropriatezza e dell'EBM e una gestione integrata dell'assistenza per una continuità individualizzata della cura del singolo paziente. Ciò richiede, da un lato, l'integrazione dei LIS con l'HIS (hospital information system) e con la rete informatica regionale e nazionale per la creazione di quello che viene definito health information technology (HIT) e health information exchange (HIE) e, dall'altro, che knowledge management e performance management sostituiscano le preoccupazione per volumi di lavoro e costi [27]. D'altra parte, la diffusione di POCT e selftesting, spesso selvaggia per l'assenza di regole e per le luddistiche resistenze di sacche retrograde di professioni sanitarie, la sinergia/sintesi di tecnologie diagnostiche appartenenti a diverse discipline di laboratorio e anche di altre aree professionali e disciplinari, la necessità di rispondere al quesito clinico con valore aggiunto in tutte le situazioni cliniche e a tutti i pazienti che ne hanno bisogno, spingono i Laboratori più avvertiti ad assumere le caratteristiche del Laboratorio ibrido, necessariamente sostenuto da un'adeguata ICT.

Le caratteristiche dell'architettura del laboratorio clinico che guarda al futuro possono essere sintetizzate in tre attributi [5]: functionality, flexibility e forwardness (le $3 \mathrm{~F}$ ). La functionality è definita come l'insieme delle funzioni eseguite o supportate dalla tecnologia. Ovviamente ciò dipende fortemente dall'automazione, dal suo disegno di sistema e dai suoi rapporti con il software, in particolare le interfacce tra il LIS e il LAS. La gestione strumentale in un LAS richiede l'implementazione di un modulo di controllo che tende a sostituire l'operatore umano con regole interiorizzate non automatiche (il tecnico di laboratorio) mediante un sistema di 
controllo automatico con regole pre-impostate che consente un pre-definito livello di operazioni ininterrotte e controllate prima di richiedere l'intervento umano. Il LIS deve a sua volta gestire, a livelli progressivamente superiori, i diversi sistemi operativi (ad esempio, POCT), integrandoli e garantendo l'omogeneità del servizio a livello della richiesta, delle connessioni strumentali e dei middleware, del controllo e della trasmissione di risultati e referti. A livello di informazioni aggregate il LIS gestisce funzioni più complesse cliniche (reporting e $R \& D$ ) e manageriali (controllo di qualità, approvvigionamenti, statistiche) rivolte all'accountability. Al più alto livello di conoscenza il LIS gestisce le funzioni di supporto alla decisione clinica e quelle gestionali di monitoraggio delle performance. Il focus di IT nel Laboratorio dovrebbe seguire i cambiamenti evolutivi e migrare dai tipici problemi interni al Laboratorio (collegamenti strumentali, trasmissione dati, ecc.), dati ormai per assodati, verso i temi più ampi del servizio esterno (integrazione di sistemi clinici, clinical audit, ecc.). Flexibility. L'aspetto essenziale per una flessibilità compiuta e prospettica è la modularità e scalabilità delle proposte tecnologiche, per essere pronti all'aumento dei volumi, alle modifiche organizzative e all'innovazione medica e assistenziale. D'altra parte la valutazione del modello organizzativo di Laboratorio più adeguato alle necessità della "popolazione naturale", come definita dalla Pathology Modernisation, deve poggiare su una base dati completa e sicura, tempestivamente aggiornata, dalla quale poter in tempo reale produrre proiezioni organizzative. Queste necessità amministrative servono contestualmente per la crescente richiesta di accountability rivolta ai manager del Laboratorio. Inoltre, il passaggio a organizzazioni più complesse che integrino i consolidamenti geografici, la diffusione dei POCT e il ruolo crescente della medicina di famiglia deve essere supportato dall'integrazione dei sistemi informatici di aziende diverse, di discipline diverse, di laboratori multipli. Futuribilità. Le scelte strumentali devono essere in grado di centrare adeguatamente gli obiettivi riorganizzativi e di preparare il Laboratorio a ogni cambiamento futuro, per quanto attiene sia la propria organizzazione interna (rete micro delle professioni e delle discipline) sia l'integrazione nella più vasta rete macro delle metaorganizzazioni. Ciò comporta grandi cambiamenti nelle attività, preparazione e visione degli operatori umani, che rappresentano un grande problema (e opportunità) nel processo di automazione del Laboratorio clinico. Alle ristrutturazioni, con diminuzione di professionisti e modifica delle competenze richieste, conducono la diminuzione dei finanziamenti dedicati e il nuovo modello automatizzato, "integrato" (ibrido) e "virtuale" di Laboratorio, ma anche la necessità di inserimento nelle nuove richieste dei sistemi di salute. In un quadro di organizzazione sanitaria che muta, saldando sempre più livelli territoriali e ospedalieri e puntando sulla de-istituzionalizzazione dei malati, in particolare dei portatori delle malattie "comuni", evidenze sempre maggiori, seppure non sempre perfettamente strutturate, mostrano il ruolo del Laboratorio nell'appropriatezza ed efficacia clinica delle cure.

Una prospettiva visionaria sull'evoluzione del Laboratorio nel 2026 è stata descritta alla metà del decennio scorso da O'Leary [23]. La partecipazione del privato sarà importante (almeno un terzo dell'attività totale), tenuto conto dell'attuale assoluta predominanza del pubblico ospedaliero nella realtà anglosassone. I nuovi Laboratori saranno costruiti in aree verdi adiacenti ai grandi assi viari, perché i pazienti esterni rappresenteranno la stragrande maggioranza delle persone servite. L'architettura hub \& spokes definirà i rapporti della rete nelle grandi aree strategiche (ben oltre il milione di abitanti). La tecnologia robotica e umanoide (nanotecnologie, sistemi microelettromeccanici, microfluidica, biosensori, chip impiantabili, identificazione in radiofrequenza, automi “intelligenti”, ecc.) sarà centrale e determinerà profondi cambiamenti professionali, con nuovi ruoli e funzioni (advanced practitioner scientist, clinical diagnostic scientist, skills mix manager, audit manager, ITC manager, ecc.). Sono già aspettative comuni l'aumento quantitativo e il miglioramento qualitativo della patologia molecolare diagnostica, basata sull'esame degli SNP (short nucleotide polymorphism), della proteomica e transcrittomica e della genomica, e lo sviluppo della citogenetica e della genomica forense. L'utilizzo e il trattamento delle cellule staminali avranno un progresso esponenziale, nelle GMP factory. Nel 2026 la cultura di organi sarà una realtà. La tassonomia delle discipline e sub-discipline di laboratorio sarà sconvolta. La Cellular Science avrà nella telepatologia e nella conseguente tele-sinergia il modello per integrare una rete europea (EUROPATH Consortium) di diagnostica cellulare, in grado di scambiare immagini digitali e report virtuali 24 ore al giorno per 7 giorni/settimana tutto l'anno. L'intelligenza artificiale aiuterà nell'individuazione e nel controllo degli eventi cellulari rari. L'integrazione delle immagini con i dati della genomica, proteomica e metabolomica migliorerà la "precisione" diagnostica sugli aspirati di un gruppo di cellule o anche di una sola cellula. La citopatologia vaginale andrà scomparendo in conseguenza della diffusione della vaccinazione HPV. Lo sviluppo della tecnologia dell'immagine (contemporanea analisi PET-TC e PET-RM; capacità di cellular e molecular imaging) entrerà in sinergia con la nuova "scienza cellulare". L'integrazione di ematologia, medicina trasfusionale, biochimica e immunolo- 
gia darà origine a una disciplina unificata, la Blood Sciences, ampiamente già oggi preparata dai Laboratori core. Accanto alla nanotecnologia, alla bio-robotica, alla miniaturizzazione più spinta dei sensori (microdevice), la creazione di chip impiantabili porterà alla definizione di micro-impianti semi-permanenti personalizzati (personal profile chip, PPC) per il monitoraggio continuo (e la somministrazione terapeutica) dei pazienti cronici (cardiopatici, oncologici, diabetici, autoimmunitari e così via), il cui controllo sarà possibile in telemetria remota. In breve questi chip saranno in grado di analizzare direttamente i geni nelle cellule e nei tessuti in vivo (phenotypic monitoring gene chips, PMGC). La microbiologia clinica subirà le più profonde innovazioni nel campo dei test di sensibilità alle terapie, del monitoraggio ambientale, dell'epidemiologia molecolare e dell' $i$ dentificazione dei ceppi e degli agenti infettivi. Le malattie infettive saranno diagnosticate ampiamente con lab-on-chip costruiti per estrazioni, identificazioni e sequenziamenti automatici e miniaturizzati. Il monitoraggio delle malattie infettive sarà effettuato nel luogo di cura, in remoto dal Laboratorio.

La prospettiva descritta può sembrare eccessiva, ma Beastall [19], facendo il punto sull'evoluzione della Pathology Modernisation nel 2008, descrive una realtà anglosassone in movimento verso quella direzione. I network di patologia continuano a svilupparsi in ragione delle economie di scala, della moderna tecnologia e delle esperienze prodotte. A livello locale il principio $h u b$ \& spokes fatica a realizzarsi, ma è il modello cui tendere. La diagnostica di laboratorio è sempre più interconnessa con la diagnostica per immagini e si procede a una loro integrazione focalizzata al paziente. Il concetto di blood science sta emergendo come una realtà operativa efficiente. Talora realtà più ampie del concetto di blood science vengono gestite da contratti di service. È in corso una ri-definizione dei ruoli degli operatori. Sempre meno persone stanno facendo il lavoro per il quale sono state preparate. Lo sviluppo professionale è un tema emergente per le leadership.

\section{Un futuro possibile}

Secondo Friedberg e Weiss [28], i trend della Medicina di Laboratorio dipendono dalle dinamiche chiave legate alla tecnologia (molecular diagnostic, molecular imaging, nanotechnology), all'IT (data mining, aggregate information, innovative thinking), agli aspetti medici della 4Ps medicine e in particolare della "preemptive medicine" e della cura di pazienti cronici nelle loro sedi e attività naturali (monitoraggio e biomarcatori) e a quelli del mercato. Da quest'ultimo punto di vista, gli Autori americani sottolineano almeno tre aspetti: la necessità di comprimere i costi legata al loro aumento per la complessità dei test e alle forme di rimborso, lo shift da pazienti prevalentemente ospedalizzati a pazienti prevalentemente ambulatoriali e l'impatto delle dinamiche industriali (merging e sinergie di aziende; aziende impegnate sia nella diagnostica sia nell'area terapeutica, tra loro collegate). In Europa si sostituirà all'importanza dei sistemi di rimborso quella del ridisegno dell'organizzazione dei servizi sanitari pubblici, in conseguenza delle ristrettezze finanziarie [3]. In ogni caso le conclusioni sono che la spinta ai consolidamenti, al taglio dei costi e all'innovazione tecnologica e organizzativa continuerà, in direzione di grandi Laboratori di riferimento con economie di scala, reingegnerizzazione dei processi, riduzione di personale e preminenza della tecnologia, in particolare quella informatica. L'unica forza contraria è l'importanza di fornire la cura a livello del paziente, ma la tecnologia potrà consentirlo senza il mantenimento dell'attuale organizzazione dei Laboratori. Le conclusioni degli autori [28] sono, peraltro, ottimistiche. Il futuro è pieno di opportunità mascherate da sfide e difficoltà: mutano le forme, ma la necessità di Diagnostica di Laboratorio andrà aumentando. Una ricerca di Frost e Sullivan [29] del 2011 sull'andamento del mercato europeo di IVD (in vitro diagnostic) prevede un aumento di circa il 70\% nel 2018 (circa 8\% per anno). I laboratoristi sono in grado di coglierle attraverso la combinazione di talento, esperienza, capacità manageriali, per mantenere e migliorare il ruolo di valore aggiunto nei processi di cura, anche se c'è preoccupazione che team decimati e invecchiati fatichino a tenere il passo dell'innovazione. Non diversamente, un'analisi italiana dello stesso anno ha sottolineato il ruolo e le caratteristiche della Medicina di Laboratorio per il miglioramento delle cure: un servizio essenziale, una prospettiva interdisciplinare e una visione olistica [30].

Un ulteriore tema che angustia i professionisti della Medicina di Laboratorio è quello di come affrontare il cambiamento. Si è preferito agire attraverso una "dynamic stability" [31], un'innovazione sostenibile, di solito evolutiva, talora rivoluzionaria, che procedesse per aggiustamenti tecnologici e organizzativi volti a conservare o migliorare il servizio al "mercato" (medici e pazienti) tradizionale e che tenesse in debito conto la naturale resistenza al cambiamento, i mandati contraddittori, la cultura mitica della "tribù" e la tendenza all "inerzia attiva" derivante dai vincoli organizzativi e logistici delle organizzazioni sanitarie. Tuttavia, oggi è maggiormente rispondente alla situazione della Medicina di Laboratorio l'approccio che Christensen [32] ha chiamato "disruptive innovation", innovazione dirompente, sconcertante, distruttiva, la creazione di un 
nuovo mercato e di una nuova rete di valore che può distruggere i precedenti, tradizionali ambiti di produzione e di vendita. Proprio Christensen ha suggerito che i POCT e l'IT sono due esempi di innovazione dirompente/distruttiva nell'ambito del Laboratorio, perché consentono (obbligano) a una medicina più "precisa", a livello sia diagnostico sia organizzativo, sconvolgendo i modi tradizionali di fornitura del servizio e i ruoli e compiti dei suoi operatori. Da riflessioni simili, Price [17] trae spunto per indicare i compiti chiave per il futuro della Medicina di Laboratorio: la garanzia della qualità, il knowledge management, la ricerca traslazionale $\mathrm{e}$ "trasformazionale" (applicazione delle innovazioni ed evidenze in pratica) e la generazione di "evidenze" utili all'uso dei nuovi test, all'impatto assistenziale del nuovo Laboratorio e alla strutturazione e finanziamento delle organizzazioni.

La riflessione centrale rispetto ai cambiamenti in corso e del prossimo futuro è quella intorno ai ruoli e alle caratteristiche degli operatori medici, scientist, tecnici nella loro specifica preparazione e nell'esperienza e attività all'interno di un'equipe coordinata e collaborativa. Alcune di queste trasformazioni sono state descritte da circa un decennio [5]. Recentemente ricercatori del CERGAS (Centro di Ricerche sulla Gestione dell'Assistenza Sanitaria e Sociale) Bocconi [33], con il supporto di Siemens Healthcare Diagnostics Italia e del suo Advisory Board, hanno proposto un'indagine volta a definire, nel quadro delle dinamiche del Servizio Sanitario Nazionale (SSN), i ruoli dei professionisti di laboratorio, le dinamiche dei servizi di laboratorio e il presidio dei saperi disciplinari. Le interconnessioni tra i livelli affrontati sono molte, forti e decisive. L'evoluzione organizzativa del SSN condiziona quella del Laboratorio tra esternalizza- zioni e concentrazioni ed entrambe determinano il prevalere dei ruoli manageriali su quelli disciplinari (o viceversa) e il bouquet dei saperi da presidiare (Fig. 2). Le possibili vocazioni del professionista di laboratorio sono individuate in: sacerdote della tecnologia (innovazione tecnologica, selezione di tecnologie e partner, controllo di qualità, valutazione delle forme di gestione); ruolo organizzativo-gestionale (pianificazione, organizzazione e gestione del Laboratorio e dei suoi addetti, con particolare attenzione ai processi, al prodotto e al loro controllo); consulente clinico (consulenza nella richiesta e nell'interpretazione dei casi singoli, gruppi interdisciplinari, audit clinico); governo clinico (linee guida, EBLM, PDTA).

\section{Ruoli e scenari al tempo della crisi}

La trasformazione del Laboratorio e della Medicina di Laboratorio sono sconvolgenti: aprono incredibili possibilità diagnostiche, ma rischiano di far scomparire disciplina e professionisti. Tutte le previsioni danno per inarrestabile il progresso verso una Medicina più "precisa" e focalizzata al "nuovo" paziente. Le organizzazioni del Laboratorio, così come le abbiamo conosciute e abbiamo tentato di difendere e far maturare attraverso dinamiche evolutive, non sono più adeguate alle nuove sfide della sanità. I professionisti della Medicina di Laboratorio, la loro educazione istituzionale e continua e i contenuti professionali di cui dispongono sono sottoposti a radicali innovazioni. Si tratta di immaginare il futuro con coraggio e con la prospettiva di saper cambiare profondamente i nostri saperi e i nostri modi di operare. Molte delle suggestioni degli Autori ricordate in questo Editoriale ci possono essere utili. Una riflessione aperta e approfondi-

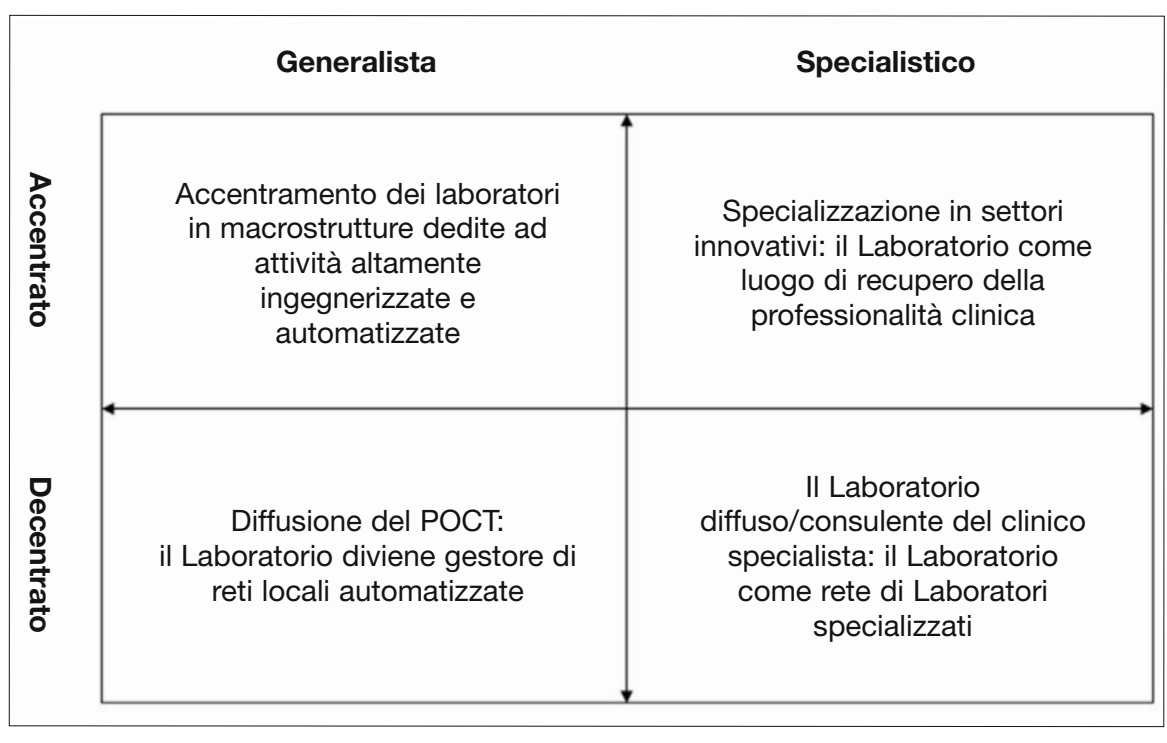

Fig. 2 La possibile evoluzione del Laboratorio secondo gli attributi generalista/specialista e accentrato/decentrato. POCT, point-ofcare testing. Mod. da Longo [33] 
ta su ruoli e scenari al tempo della crisi è necessaria. Nel frattempo, alcuni strumenti concettuali e operativi per affrontare il futuro sono stati già proposti nel "Patto per la Modernizzazione e l'Umanizzazione della Medicina di Laboratorio in Italia"[34], che mantiene ancora oggi il suo valore di visione e di proposta. Per rispondere adeguatamente alla sua natura, "fornire informazioni cliniche", la Medicina di Laboratorio deve organizzarsi in una "rete ("micro" nei rapporti interprofessionali e interdisciplinari dell'equipe e "macro" nei rapporti tra organizzazioni che servono la popolazione "naturale") gestita (non si tratta di una rete da pescatore, ma di una tela di ragno) di "servizi" (non semplicemente prestazioni o test), per governare l'intero processo che la origina, attiva e utilizza. Il punto chiave diventa l'interfaccia clinica-laboratorio, amplificata a dismisura dalla struttura a rete, rivolta al clinico e al paziente, coinvolgente tutta l'equipe, caratterizzata dalla qualità della comunicazione e dalla sua coerenza con l'obiettivo clinico. Tutto ciò si realizza secondo le possibilità tecnologiche e le sensibilità culturali. I due aspetti, organizzativo e concettuale, vanno fusi in un approccio globale, nel quale il servizio della Medicina di Laboratorio e il suo impatto sulla cura dei pazienti vengono declinati nella rete dei rapporti che ne costituiscono la popolazione "naturale", secondo modalità congrue al concetto di centralità del paziente e di appropriatezza dell'intervento. L'applicazione di una Medicina di Laboratorio così concepita deve fronteggiare numerosi problemi, quali la pluralità dell'utente finale, medico curante e cittadino, l'intersecarsi dei piani di azione generali o rivolti al singolo paziente, la molteplicità delle nuove competenze richieste e, soprattutto, la consapevolezza che l'affidabilità della fase analitica non è più un sicuro rifugio per una disciplina costretta ad aprirsi al mondo clinico e alla società.

Conflitto di interessi Nessuno

\section{Bibliografia}

1. Parker KM, Talbert ML (2003) Laboratory Medicine in the 21th Century. In Ward-Cook KM, Lehmann CA, Scoeff LE, Williams RH Eds. Clinical Diagnostic Technology. The Total Testing Process. Volume 1: The Preanalytical Phase. Washington: AACCPress

2. Cappelletti P (2012) Medicina di Laboratorio e Postmodernità. Riv Ital Med Lab 8:1-15

3. Cappelletti P (2012) Il futuro della Sanità: sostenibilità finanziaria e scenari evolutivi. Riv Ital Med Lab 8:63-70

4. Moving toward precision medicine (2011) The Lancet 378:1678

5. Cappelletti P (2010) Medicina di Laboratorio. In Galzigna L, Plebani M (a cura di). Trattato Italiano di Medicina di Laboratorio. Volume I Biochimica Clinica Generale. Padova: Piccin Nuova Libraria

6. Cappelletti P (2004) Un patto per la modernizzazione della
Medicina di Laboratorio in Italia. Riv Med Lab - JLM 5 Suppl 3:17-32

7. Markin RS, Whalen SA (2000) Laboratory automation: traiectory, technology, and tactics. Clin Chem 46:764-771

8. Burke MD (2000) Laboratory medicine in the 21st Century. Am J Clin Pathol 114:841-846

9. Kiechle FL, Main RI (2002) Improving efficiency in the Clinical Laboratory. Washington: AACC Press

10. Burtis CA (1996) Converging technologies and their impact on clinical laboratory. Clin Chem 42:1735-1749

11. Hoffmann GE (1998) Concepts for the third generation of laboratory systems. Clin Chim Acta 278:203-216

12. Felder RA (1998) Modular workcells: modern methods for laboratory automation. Clin Chim Acta 278:257-267

13. Goldberg DM, Diamandis EP (1994) Clinical Chemistry: Death or Transfiguration. Eur J Lab Med 2:157-159

14. Panteghini M (2004) The future of Laboratory Medicine: understanding the new pressure. Clin Biochem Rev 25:207-215

15. Plebani M (2005) The future of clinical laboratories: more testing or knowledge services? Clin Chem Lab Med 43:893-896

16. Bossuyt X, Verweire K, Blanckaert N (2007) Laboratory Medicine: Challenges and Opportunities. Clin Chem 53:1730-1733

17. Price CP (2010) Roots, development and future directions of laboratory medicine. Clin Chem Lab Med 48:903-909

18. Michel RL (2003) Hot Interview on Laboratory Management. http://www.darkreport.org [Accesso 30 luglio 2012]

19. Beastall GH (2008) The Modernisation of Pathology and Laboratory Medicine in the UK: Networking into the Future. Clin Biochem Rev 29:3-9

20. Langlois MR, Wellemacq P (2009) The future of hospital laboratories. Position statement from the Royal Belgian Society of Clinical Chemistry (RBSCC). Clin Chem Lab Med 47:1195-1201

21. Cappelletti P (2008) Come cambia la Medicina di Laboratorio. RIMeL/IJLaM 4(Suppl):26-30

22. Barth J (2010) The future for laboratory medicine. The Bulletin of the Royal College of Pathologists 151:182-183. http://www.rcpath.org [Accesso 30 luglio 2012]

23. O'Leary JJ (2006) Pathology 2026: The future of laboratory medicine and academic pathology. In O'Leary JJ. Understanding disease. New York: John Wiley \& Sons

24. Cappelletti P (2003) Metaplan ${ }^{\circledR}$ : un nuovo metodo di diagnosi e cura dei mali della Medicina di Laboratorio. Riv Med Lab - JLM $5: 249$

25. Friedman BA (2001) The total laboratory solution: a new laboratory e-business model based on a vertical laboratory meta-network. Clin Chem 47:1526-1535

26. Kost GJ (1992) The hybrid laboratory: the clinical laboratory in the 1990s is a synthesis of the old and new. Arch Pathol Lab Med 116: $1002-1003$

27. Dorizzi RM (2007) Una roadmap per la Information Technology in Medicina di Laboratorio. RIMeL/IJLaM 3:75-81

28. Friedberg RC, Weiss RL (2007) Future Trends. Clin Lab Med 27:931-936

29. http://www.frost.com/prod/servlet/report-toc.pag?repid=M7C801-00-00-00 [Accesso 30 luglio 2012]

30. Cappelletti P (2007) La Medicina di Laboratorio ed il miglioramento delle cure: un servizio essenziale, una prospettiva interdisciplinare, una visione olistica. RIMeL/IJLaM 3(Suppl):19-24

31. Abrahamson E (2000) Change without pain. Harv Bus Rev 4:75-79

32. Christensen CM, Overdorf M (2000) Meeting the challenge of disruptive change. Harv Bus Rev 2:66-76

33. Longo F (2011) Scenari per la sanità futura: quale strategia di ruolo per i dirigenti di laboratorio? In Scenari sulla trasformazione del capitale umano. Workshop Siemens Healthcare Diagnostics. $1^{\circ}$ Congresso Nazionale della Medicina di 
Laboratorio, Parma 15-18 novembre 2011. http://www.congressomedicinalaboratorio.it/images/stories/pdf/A11_007_Programm aDefinitivo.pdf [Accesso 30 luglio 2012]
34. Cappelletti P (2004) Un Patto per la Modernizzazione e la Umanizzazione della Medicina di Laboratorio in Italia. Riv Med Lab - JLM 5:252-254 